\title{
Admittance and Permittivity in Doped Layered TlGaSe 2 Single Crystals
}

\author{
S.A. Dawood ${ }^{a}$, A.K. Fedotovi, ${ }^{a,}$ T.G. Mammadov ${ }^{b}$, P. Zukowski ${ }^{c}$, T.N. Koltunowicz ${ }^{c}$, \\ A.M. SAAD ${ }^{d}$ AND N.A. DROZDOV ${ }^{a}$ \\ ${ }^{a}$ Belarusian State University, Independence av. 4, 220030 Minsk, Belarus \\ ${ }^{b}$ Institute of Physics of Azerbaijan National Academy of Science, Baku, Azerbaijan \\ ${ }^{c}$ Department of Electrical Devices and High Voltages Technologies, Lublin University of Technology \\ Nadbystrzycka 38a, 20-618 Lublin, Poland \\ ${ }^{d}$ Al-Balqa Applied University, P.O. Box 4545, Amman 11953, Jordan
}

\begin{abstract}
In doped $\mathrm{TlGaSe}_{2}$ crystals the phase transitions at low temperatures $(100-170 \mathrm{~K})$ were observed using admittance and dielectric spectroscopy in a temperature range of 80-320 K. The admittance and permittivity measurements in the studied samples indicated that after Fe or Tb doping by impurities with concentrations $N_{\text {imp }}<0.5$ at.\% nonequilibrium electronic phase transition is observed. Doping with $N_{\mathrm{imp}}>0.5$ at.\% resulted in full suppression of this phase transition presence.
\end{abstract}

DOI: $10.12693 /$ APhysPolA.125.1267

PACS: 73.61.Ey, 84.37.+q, 64.70.Nd

\section{Introduction}

The ternary compound $\mathrm{TlGaSe}_{2}$ belongs to the group of chalcogenide semiconductor crystals with layered structure which are crystallized in monoclinic structure. These crystals draw a great attention due to their optical and electrical properties in view of the possible optoelectronic device application. In recent years, there is a growing interest in their study due to the coexistence of ferroelectric and semiconducting properties, as well as the presence of structural phase transitions (PTs) in some temperature ranges. In particular, it has been established that undoped $\mathrm{TlGaSe}_{2}$ crystals exhibit on cooling a sequence of structural PTs which are accompanied by peculiarities in temperature dependences of electric, dielectric, elastic, acoustic, thermal, optical, etc. properties at temperature ranges of about $90-140 \mathrm{~K}$ and $240-260 \mathrm{~K}$ [1-6]. A great number of experimental results indicated that in undoped $\mathrm{TlGaSe}_{2}$ a sequence of low-temperature structural PTs from a paraphase to an incommensurate phase at $T_{\mathrm{i}} \approx 120 \mathrm{~K}$ and then to the commensurate ferroelectric phase at $T_{\mathrm{c}} \approx 110 \mathrm{~K}$ are observed. The ferroelectric $\mathrm{PT}$ is a displacive improper type and the spontaneous polarization at $T \approx 110 \mathrm{~K}$ is inside the layer planes. So it was interested to check the effect of doping on these PTs and their manifestations using dielectric and impedance spectroscopy.

The present paper is devoted to the study of AC electric and dielectric properties at different temperatures and frequencies in $\mathrm{TlGaSe}_{2}$ crystals doped with different

\footnotetext{
*corresponding author; e-mail: fedotov@bsu.by
}

impurities and their comparison with the properties of the undoped crystals.

\section{Experimental}

The single crystals were grown and doped with Fe, $\mathrm{Ag}, \mathrm{B}, \mathrm{Tb}, \mathrm{Er}$, and $\mathrm{Al}$ impurities in evacuated quartz tubes by using the modified Bridgman method [5-8]. The studied samples with rectangular form were oriented by larger face (the cleavage plane) along the polar axis. Scanning electron microscope images and stoichiometry of the grown single crystals were shown in [8] earlier. The Raman spectra were taken at room temperature using a Nanofinder High End (Lotis TII, Belarus-Japan) confocal microscope-based setup. The Raman scattering was excited using the $532 \mathrm{~nm}$ solid-state laser with power level at the sample at $c a .60 \mu \mathrm{W}$ to minimize laser-induced damage to the crystals. The spectral calibration was done using a built-in gas-discharge lamp with accuracy better than $1 \mathrm{~cm}^{-1}$. Transmission spectra of the crystals were measured using a spectrophotometer MC 122 (Proscan Special Instruments, Belarus) in a spectral range $200-1100 \mathrm{~nm}$.

Temperature/frequency dependences of the real and imaginary parts of admittance and permittivity were measured on the samples of $4-5 \mathrm{~mm}$ width, 5-6 mm length and $0.5-0.8 \mathrm{~mm}$ thickness. For measurements the wide faces of samples were gently polished, cleaned and covered with two indium probes, which were applied by ultrasound soldering, so that the measuring current was carried normally to the best cleavage plane (normally to $c$ axis). Admittance and dielectric spectroscopy of the samples was studied by means of a 2-probe method in a temperature range of $80-340 \mathrm{~K}$. Measurement setup included either a precise HIOKI 3532 LCR HiTESTER 
AC bridge (for the frequency range $50 \mathrm{~Hz}-100 \mathrm{kHz}$ ) or Hewlett Packard LCR-meter HP 4284A (for measurements at frequencies $100 \mathrm{kHz}-5 \mathrm{MHz}$ ) was used. During the experiments, the changes in the full resistance and phase of the samples studied were fixed to calculate the real and imaginary parts of impedance and permittivity. The absolute values of admittance and permittivity were measured accurate up to 4\%. All AC measurements were carried out with a full computer control.

\section{Results and discussion}

Scanning electron microscopy (SEM) investigations of single crystals confirmed their stoichiometry and have shown that surfaces of their normally-cleavaged faces displayed layered-like structure with either piramid- and step-like roughnesses (see [8] and insets 1 and 2 in Fig. 1a) or dendritic and brushy inclusions (insets 3 and 4 in Fig. 1b) with about 1-2 $\mu \mathrm{m}$ sizes. Let us note that, as was mentioned in [8], some of these inclusions were enriched by basic components of the crystals (mainly selenium or thallium).
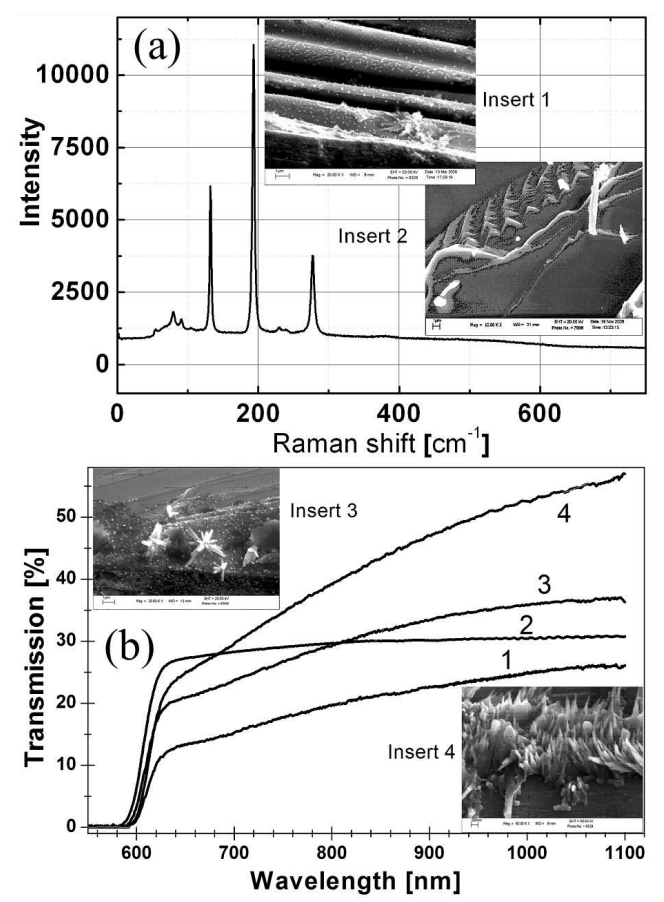

Fig. 1. Raman (a) and transmission (b) spectra for $\mathrm{TlGaSe}_{2}$ crystal doped with $\mathrm{Tb}$ (1), Fe (2), Al (3) and Ag (4). SEM images show surfaces of cleavaged plates (inserts 1 and 2) and natural faces (inserts 3 and 4) of crystals after growth.

The examples of Raman and reflectance spectra for the doped $\mathrm{TlGaSe}_{2}$ crystals are presented in Fig. 1 . The spectral position of the observed the Raman lines (Fig. 1a) corresponds to the values known from the literature for undoped $\mathrm{TlGaSe}_{2}$ crystals $[9,10]$. Moreover, the parameters of Raman lines (both position and FWHM) do not depend on the type and concentration of the studied dopants indicating no detectable influence of the doping on the lattice properties.

Transmission curves $\alpha(\lambda)$ in Fig. 1b for the differently doped crystals display similar behavior in the low wavelength values (between 580 and $640 \mathrm{~nm}$, i.e. in the vicinity of absorption edge). Re-plotting $\left(\alpha_{\lambda} h \omega\right)^{2}$ as a function of $h \omega$ for $h \omega \geq E_{\mathrm{g}}(\omega-$ circular frequency), we evaluated the energy gap $E_{\mathrm{g}} \approx 2.077 \mathrm{eV}$ which conforms with the value known from literature [1, 10]. Influence of doping on absorption edge was also not observed. We attribute differences in $\alpha(\lambda)$ curves beyond the absorption edge in Fig. 1b to different state of the surface of the crystalline plates studied.

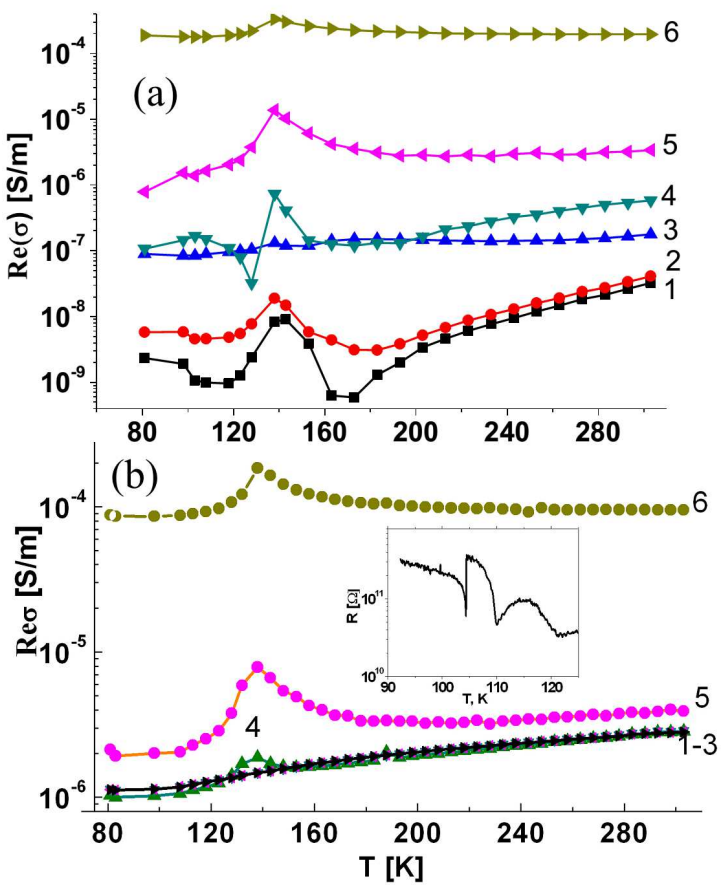

Fig. 2. Temperature dependences of real part of admittance $\operatorname{Re} \sigma(T)$ for $\mathrm{TlGaSe}_{2}$ crystals doped with 0.3 at.\% $\mathrm{Fe}(\mathrm{a})$ and 0.1 at.\% $\mathrm{Tb}$ (b) measured at different frequencies: $1-f=500 \mathrm{~Hz}, 2-1 \mathrm{kHz}, 3-$ $10 \mathrm{kHz}, 4-100 \mathrm{kHz}, 5-1 \mathrm{MHz}, 6-3 \mathrm{MHz}$. Inset presents temperature dependence of DC resistance in low-temperature range observed in [8] for $\mathrm{TlGaSe}_{2}\langle\mathrm{Fe}\rangle$ crystal.

The doping of crystals has resulted in the lowering of their resistivity as compared to the undoped ones $[2,11,12]$ that was direct indicator of the doping influence on the properties of the studied crystals and allowed to study AC/DC conductance down to $80 \mathrm{~K}$. In order to get additional information about the succession of PTs in the doped $\mathrm{TlGaSe}_{2}$ crystals versus undoped ones, the frequency dependences of the admittance and permittivity in the above mentioned temperature region were measured.

The examples of temperature dependences of real and imaginary parts of admittance and permittivity for 


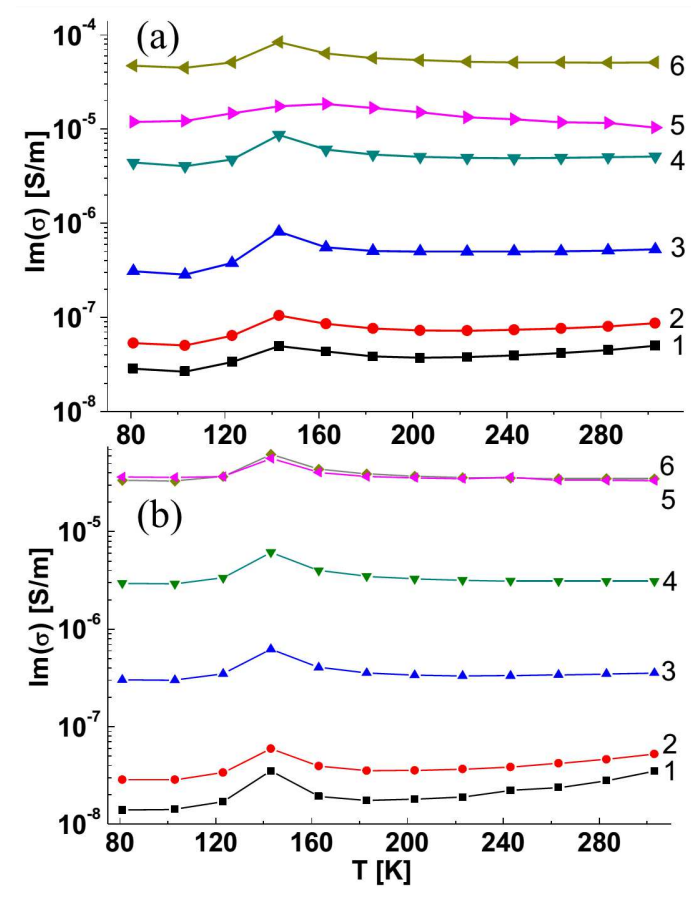

Fig. 3. Temperature dependences of imaginary part of admittance $\operatorname{Im} \sigma(T)$ for $\mathrm{TlGaSe}_{2}$ crystals doped with 0.3 at.\% $\mathrm{Fe} \mathrm{(a)}$ and 0.1 at.\% $\mathrm{Tb}$ (b) measured at different frequencies: $1-f=500 \mathrm{~Hz}, 2-1 \mathrm{kHz}, 3-$ $10 \mathrm{kHz}, 4-100 \mathrm{kHz}, 5-1 \mathrm{MHz}, 6-3 \mathrm{MHz}$.

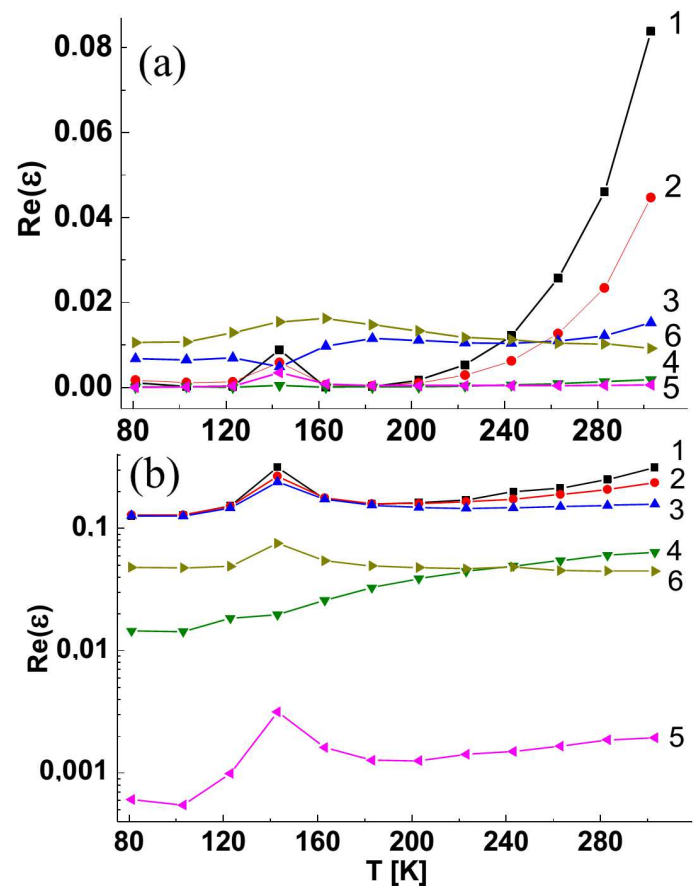

Fig. 4. Temperature dependences of real part of permittivity $\operatorname{Re} \varepsilon(T)$ for $\mathrm{TlGaSe}_{2}$ crystals doped with 0.3 at.\% $\mathrm{Fe}$ (a) and 0.1 at.\% $\mathrm{Tb}$ (b) measured at different frequencies: $1-f=500 \mathrm{~Hz}, 2-1 \mathrm{kHz}, 3-$ $10 \mathrm{kHz}, 4-100 \mathrm{kHz}, 5-1 \mathrm{MHz}, 6-3 \mathrm{MHz}$.
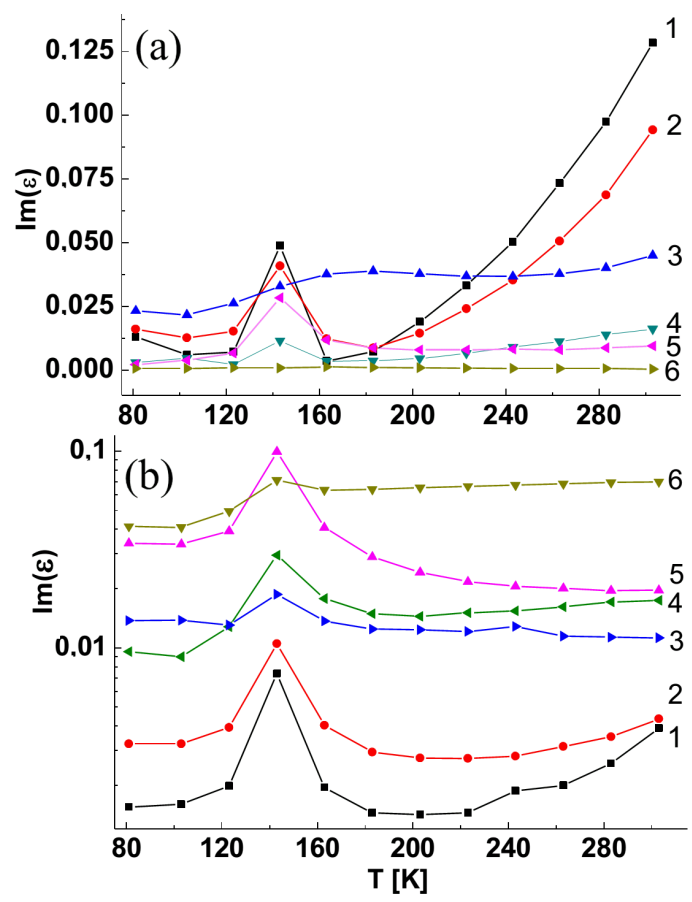

Fig. 5. Temperature dependences of imaginary part of permittivity $\operatorname{Im} \varepsilon(T)$ for $\mathrm{TlGaSe}_{2}$ crystals doped with 0.3 at.\% $\mathrm{Fe} \mathrm{(a)}$ and 0.1 at.\% $\mathrm{Tb}(\mathrm{b})$ measured at different frequencies: $1-f=500 \mathrm{~Hz}, 2-1 \mathrm{kHz}, 3-$ $10 \mathrm{kHz}, 4-100 \mathrm{kHz}, 5-1 \mathrm{MHz}, 6-3 \mathrm{MHz}$.

the samples of doped $\mathrm{TlGaSe}_{2}\langle\mathrm{Fe}\rangle$ and $\mathrm{TlGaSe}_{2}\langle\mathrm{~Tb}\rangle$ crystals are presented in Figs. 2-5. As is seen, both samples, doped with low concentration of $\mathrm{Fe}$ and $\mathrm{Tb}$ $\left(N_{\text {imp }}<0.5\right.$ at.\% $)$ show clearly expressed peculiarities in $\operatorname{Re} \sigma(T)$ and $\operatorname{Im} \sigma(T)$ dependences in the temperature range 100-170 K (Figs. 2 and 3). However in the sample $\mathrm{TlGaSe}_{2}\langle\mathrm{Fe}\rangle$ the character of $\operatorname{Re} \sigma(T)$ progress strongly depends on frequency displaying two types of peculiarities shape. The 1st type peculiarity for $f<10 \mathrm{kHz}$ displays both maximum and minimum on $\operatorname{Re} \sigma(T)$ curves 1 and 2 in Fig. 2a, whereas for higher frequencies we observed the 2nd type of $\operatorname{Re} \sigma(T)$, curves 5 and 6 , which are characterized by only maximum. For $\mathrm{TlGaSe}_{2}\langle\mathrm{~Tb}\rangle$ samples we observed only peculiarity of the 2nd type (Fig. 2b). It is necessary also to note that doping by non-magnetic impurities ( $\mathrm{Al}, \mathrm{Ag}, \mathrm{B})$ did not result in such clearly expressed peculiarities of $\mathrm{AC}$ response in the above temperature range. Moreover, at doping with $N_{\text {imp }}>0.5-1.0$ at.\% (including $\mathrm{Fe}$ and $\mathrm{Tb}$ ) we did not observe low-temperature peculiarities at all. As is seen from Figs. 4 and $5, \operatorname{Re} \varepsilon(T)$ and $\operatorname{Im} \varepsilon(T)$ dependences also show low-temperature maxima for $\mathrm{Fe}$ - and $\mathrm{Tb}$-doped $\mathrm{TlGaSe}_{2}$ crystals.

Analysis of the observed $T / f$ dependences both for admittance and permittivity show that temperatures, where PT is observed, take very wide range $100-170 \mathrm{~K}$, although maximum in all curves (if it is observed at all) lies at nearly constant temperature $140 \pm 1 \mathrm{~K}$. Let us note 
that this temperature range engrosses the known interval $110-120 \mathrm{~K}$ with maxima in $\operatorname{Re} \varepsilon(T), \operatorname{Re} \sigma(T)$ and thermal expansion coefficient at about $112-115 \mathrm{~K}$, where commensurate (C)-incommensurate (I) PT was observed in the undoped crystals $[2,5,6,11,12]$. Note, that C-I PT was also fixed first in $\mathrm{TlGaSe}_{2}$ crystals low-doped with $\mathrm{Fe}$ (less than 0.1 at.\%) by temperature dependences of DC conductivity (see inset in Fig. 2b [8]) measured along the cleavage plane (along $c$ axis). On the other side, the position of maxima $T_{\max }$ on temperature dependences of permittivity and admittance in Figs. $2-5$ is shifted to $\approx 141 \mathrm{~K}$ as compared to $T_{\max } \approx 120 \mathrm{~K}$ in $\mathrm{C}-\mathrm{I} \mathrm{PT}$ in undoped crystals. Formally, position of $T_{\max } \approx 141 \mathrm{~K}$ is very close to the beginning of non-equilibrium electronic PT due to polarization in the direction perpendicular to the layers plane that was observed in [12]. However the shape of $\operatorname{Re} \varepsilon(T), \operatorname{Re} \sigma(T)$ curves in [12] does not match with the same dependences in Figs. 2-5 for the doped crystals (especially for temperatures higher than $200 \mathrm{~K}$ ). Therefore for $\mathrm{TlGaSe}_{2}$ crystals weakly doped with $\mathrm{Fe}$ or $\mathrm{Tb}$ we can attribute with more higher probability to commensurate-incommensurate phase transition the observed low-temperature maximum on $\operatorname{Re} \varepsilon(T)$ and $\operatorname{Re} \sigma(T)$ dependences in Figs. 2-5. Possible contribution of non-equilibrium electronic PT in this shift of $T_{\max }$ demands additional experiments.

\section{Resume}

In $\mathrm{TlGaSe}_{2}$ crystals weakly doped with $\mathrm{Fe}$ and $\mathrm{Tb}$ (with $N_{\text {imp }}<0.5$ at.\%), using method of temperature dependences of admittance $\sigma(T)$ and permittivity $\varepsilon(T)$ in the temperature range of $80-300 \mathrm{~K}$, the shift of commensurate-incommensurate phase transition to the temperatures of the order of $141 \mathrm{~K}$ (as compared with $\approx 120 \mathrm{~K}$ in undoped crystals) was observed. More heavy doping of the crystals with $N_{\text {imp }}>0.5$ at.\% resulted in full suppression of any anomalies (phase transitions) on $\varepsilon(T)$ and $\sigma(T)$ dependences.

\section{Acknowledgments}

The work was partially supported by the VISBY Program of the Swedish Institute and Belarusian Fundamental Research Foundation by contracts No $\Phi 09 \mathrm{Az}-006$. Dr. T.N. Koltunowicz is a participant of the project: "Qualifications for the labour market - employer friendly university", cofinanced by European Union from European Social Fund.

\section{References}

[1] A.M. Panich, J. Phys. Condens. Matter 20, 293202 (2008).

[2] E. Şenturk, L. Tumbek, F. Salehli, F.A. Mikailov, Cryst. Res. Technol. 40, 248 (2005).

[3] F.A. Mikailov, E. Basaran, T.G. Mammadov, M.Yu. Seyidov, E. Senturk, Physica B 334, 13 (2003).

[4] F.A. Mikailov, E. Basaran, E.S. Entu, L. Tu. Mbek, T.G. Mammadov, V.P. Aliev, Phase Transit. 76 , 1057 (2003).

[5] N.A. Abdullayev, T.G. Mammadov, R.A. Suleymanov, Phys. Status Solidi B 242, 983 (2005).

[6] M.Yu. Seyidov, R.A. Suleymanov, Phys. Solid State 50, 1219 (2008).

[7] M.Yu. Seyidov, R.A. Suleymanov, T.G. Mammadov, A.K. Fedotov, S.S. Babayev, G.M. Sharifov, Jpn. J. Appl. Phys. 50, 05FD07-2 (2011).

[8] A.K. Fedotov, M.I. Tarasik, T.G. Mammadov, I.A. Svito, P. Zhukowski, T.N. Koltunowicz, M.Yu. Seyidov, R.A. Suleymanov, V. Grivickas, V. Bicbaevas, Electrical Review 88, 301 (2012), (in Polish).

[9] K. Allakhverdiev, R. Sardarly, F. Wondre, J.F. Ryan, Phys. Status Solidi B 88, K5 (1978).

[10] S.N. Mustafayev, V.A. Aliev, M.M. Asadov, Sov. Solid State Phys. 40, 561 (1998).

[11] M.Yu. Seyidov, R.A. Suleymanov, Y. Bakis, F. Salehly, J. Appl. Phys. 108, 074114 (2010).

[12] F. Salehli, Y. Bakis, M.Yu. Seyidov, R.A. Suleymanov, Semiconductor Sci. Technol. 22, 843 (2007). 